\title{
Linguistique contrastive et morphologie : les noms en -iste dans une approche onomasiologique
}

\author{
*Cartoni, Bruno \& **Namer, Fiammetta \\ *Département de Linguistique, Université de Genève, Suisse \\ Bruno.Cartoni@unige.ch \\ **UMR 7118 ATILF \& Université de Lorraine, France \\ Fiammetta.Namer@univ-nancy2.fr
}

\section{Introduction}

Pour étudier la formation morphologique des lexèmes dans une langue donnée, le morphologue n'a souvent pas d'autres choix que de se fonder sur une approche sémasiologique, c'est-à-dire de récolter l'ensemble de formes potentiellement construites par le ou les exposants du procédé à analyser. Une telle approche centrée sur le procédé de construction comporte un inconvénient majeur : parce qu'elle aborde l'analyse morphologique du point de vue de la forme des dérivés, les conclusions auxquelles elle aboutit ne lui permettent pas de rendre compte de façon exhaustive de la variété sémantique des unités produites. Et, de la même façon, une telle approche est incapable d'appréhender le lexique construit à partir de ses propriétés sémantiques. En d'autres termes, si une règle morphologique de suffixation, étudiée à partir de l'ensemble des formes collectées marquées par ce suffixe, produit majoritairement des noms d'agent, elle ne dit rien de la contribution des autres moyens de construction formant des noms s'intégrant dans la même classe référentielle.

Une manière de corriger partiellement cet inconvénient est de prendre appui sur la comparaison avec une autre langue proche, en utilisant les méthodes de la linguistique contrastive. Ces méthodes consistent à explorer de manière systématique les divergences et les similitudes entre les langues. Bien que traditionnellement associée à des finalités applicatives, que ce soit en lexicographie bilingue ou dans l'apprentissage d'une langue seconde, nous montrons que la linguistique contrastive peut également se révéler une aide précieuse dans la description de phénomènes morphologiques monolingues, en apportant à celle-ci l'éclairage onomasiologique qui lui manque.

Pour étayer notre démonstration, nous avons choisi d'étudier en parallèle deux procédés de formation supposément très similaires et observables dans deux langues proches (i.e. l'italien et le français) : la construction par suffixation de noms en -ista en italien (désormais Xista) et celle en -iste en français (désormais Xiste). Ces procédés de formation sont à première vue homogènes d'un point de vue interlingue, mais présentent néanmoins des disparités intéressantes qui apportent un éclairage nouveau sur les différentes contraintes régissant la ou les règles morphologiques opérant dans chaque langue.

Comme nous allons le voir, cette étude permet d'une part de valider (et parfois de compléter) les résultats des études monolingues, mais également d'explorer d'autres moyens (morphologiques ou non) qui permettent de nommer des agents humains. En outre, les données utilisées conduisent à une estimation des types sémantiques les plus représentés dans les formations en Xista/Xiste, ce qui offre un facteur de comparaison des sphères sémantiques dans lesquelles chaque procédé morphologique opère de manière préférentielle dans chacune des langues étudiées. Enfin, intrinsèquement, cette étude permet de questionner l'aspect prétendument translingue de la règle de suffixation en -ist $(a, e)$, et de contribuer au débat portant sur les frontières entre syntaxe et morphologie en matière de construction de lexèmes.

L'article est organisé comme suit : la section 2 est consacrée à la présentation de l'approche contrastive que nous adoptons et des données contrastives utilisées ; ensuite (section 3) nous décrivons l'état actuel des connaissances sur les procédés de formation des noms en -iste en français et en -ista en italien, en résumant deux études récentes sur le sujet. La section 4 présente les mécanismes de juxtaposition et de classification des données utilisées pour cette étude. Dans les dernières parties (sections 5 et 6), nous 
exploitons les résultats quantitatifs obtenus afin d'analyser les différences et les similitudes que présentent les deux modes de construction, en français et en italien, puis nous montrons comment l'éclairage de l'italien contribue à l'amélioration des connaissances du procédé de formation des noms en -iste du français.

\section{L'approche contrastive}

Les approches contrastives consistent à étudier de manière systématique un phénomène linguistique dans deux (ou plusieurs) langues en parallèle (en contraste) pour en faire ressortir les divergences et les similitudes (James, 1980 ; Fisiak, 1983). Souvent à visée pratique (lexicographie, enseignement de la langue seconde, traduction), l'analyse contrastive permet également d'apporter un éclairage nouveau sur un phénomène particulier dans une des deux langues en contraste (Johansson, 2007). Traditionnellement, l'approche contrastive s'effectue en trois étapes: premièrement, l'on procède à une description monolingue des phénomènes étudiés. Cette étape consiste bien souvent en la synthèse des descriptions existantes dans les langues concernées. Une telle synthèse pour les procédés de construction qui nous intéressent ici est présentée à la section 3. Dans un deuxième temps, on procède à la juxtaposition des données des deux langues, étape qui doit se faire de manière la plus objective possible (cf. ci-dessous). Enfin, la troisième étape, dite de comparaison proprement dite, consiste en l'analyse les données selon un certain nombre de critères qualitatifs et quantitatifs, permettant de mettre en lumière divergences et similitudes.

\subsection{L'analyse contrastive de la morphologie}

De tout temps, les linguistes ont fait appel, de manière plus ou moins régulière, à la comparaison interlangues pour étayer leur propos. Concernant plus précisément la comparaison inter-langues des affixes hérités du latin, ils ont fait l'objet de nombreux travaux de comparatistes. Cependant, l'étude contrastive de la morphologie, fondée sur l'analyse systématique de grands corpus, est un domaine de recherche relativement récent (cf. Lefer (2011) qui recense et évalue environ 70 études menées de 1960 à 2010 en morphologie contrastive). Parfois basée sur des groupes d'affixes, comme dans (Devos \& Taeldeman, 2004) ou (Andor, 2005), elle permet de mettre en lumière certaines contraintes qui s'exercent lors de l'application de règles dans l'une des deux langues en contraste. Plus que n'importe quel autre niveau de description linguistique, la morphologie contrastive doit se fonder sur des données réelles, et ne peut se limiter à l'intuition ou à des descriptions linguistiques. Ainsi, pour étudier une règle de construction des lexèmes d'un point de vue interlingue, il est important de récolter les sorties (output) de ces règles dans les langues analysées.

La récolte de données contrastives pour la juxtaposition constitue un défi important, à cause de la dimension interlingue, qui nécessite la mise en évidence de correspondances entre des lexèmes construits équivalents, pour ensuite analyser en contraste les procédés morphologiques ayant construit ces lexèmes. Pour ce faire, l'analyse contrastive peut se baser sur des données extraites de corpus parallèles (de traduction), permettant d'extraire plus ou moins automatiquement des paires de mots construits étant censés véhiculer le même sens. Une approche sur corpus parallèles, comme celle décrite dans (Cartoni \& Lefer, 2011) permet l'appariement des mots construits d'une langue et de tous leurs équivalents de traduction, et ainsi de rendre compte de manière exhaustive de toutes les différentes possibilités d'équivalence de traduction, qu'elles soient morphologiques ou non.

En revanche, les études contrastives basées sur corpus parallèles sont évidemment limitées. Non seulement, ce type de ressource n'est pas forcément disponible pour toutes les paires de langues, mais les corpus parallèles sont également restreints en termes de couverture du phénomène analysé : le nombre de mots construits analysés dépend intrinsèquement de la taille du corpus, et le type de construction morphologique repéré dépend également du registre ou du domaine sur lequel portent les textes du corpus. 
L'étude que nous présentons dans cet article exploite un dictionnaire bilingue comme source de données contrastives. Ce type de ressources présente des limitations plus qu'évidentes mais il offre un certain nombre d'avantages par rapport aux corpus parallèles, permettant une première approche tout à fait intéressante, comme expliqué dans la suite.

\subsection{Les dictionnaires bilingues comme sources de données contrastives}

Dans l'idéal, les études en morphologie puisent dans les ressources textuelles numérisées les données, les exemples et les contextes dont elles ont besoin pour établir ou vérifier leurs hypothèses (cf . Fradin et al., 2008 ; Hathout et al., 2009). Le morphologue a également recours aux dictionnaires monolingues, pour amorcer son expérimentation par une liste d'entrées attestées, ou appuyer son argumentation au moyen d'exemples enregistrés.

Dans cette étude, nous présentons une approche basée sur un dictionnaire bilingue. Malgré de nombreux désavantages, le dictionnaire bilingue présente, pour le chercheur, l'avantage de pouvoir récolter de manière rapide un grand nombre de données bilingues, lui permettant de couvrir un nombre relativement grand (et possiblement plus exhaustif) de phénomènes que ne le permettrait un corpus parallèle.

Évidemment, l'utilisation des dictionnaires bilingues présentent un grand nombre de biais dont il faut tenir compte dans les résultats. Les deux parties des dictionnaires bilingues $(\mathrm{L} 1 \rightarrow \mathrm{L} 2$ et L2 $\rightarrow$ L1) sont construites dans une double optique (Atkins, 2002). Par exemple, pour la section L1 $\rightarrow$ L2, le but est à la fois d'assurer une tâche de décodage (permettre à un locuteur de L2 de comprendre un énoncé en L1) et une tâche d'encodage (permettre au locuteur de L1 d'encoder en L2, c'est-à-dire de produire un message écrit ou oral en L2). Cette double tâche (répétée dans l'autre sens dans la section L2 $\rightarrow$ L1) nécessite l'inclusion d'un certain nombre d'informations qui peuvent paraître redondantes ou inappropriées pour certains utilisateurs, et pertinentes pour d'autres. Cette double articulation implique également que le sens $\mathrm{L} 1 \rightarrow \mathrm{L} 2$ n'est pas le miroir inversé du L2 $\rightarrow \mathrm{L} 1$. Un dictionnaire bilingue contient donc deux dictionnaires distincts, ce qui peut constituer une double source de données intéressante pour l'étude contrastive de la morphologie, comme nous le verrons dans la suite.

De plus, la constitution des dictionnaires bilingues repose rarement sur des corpus (parallèles ou comparables). Les données utilisées proviennent des fonds lexicographiques monolingues constitués par les maisons d'édition. En revanche, ils présentent un intérêt évident quand à leur couverture. Généralement, les entrées d'un dictionnaire bilingue sont les reflets des entrées de leurs pendants monolingues (en tout cas pour ce qui concerne les vedettes). Les traductions proposées sont normalement attestées, et vérifiées. Elles restent néanmoins le reflet des choix des lexicographes, et doivent être considérées avec toutes les précautions nécessaires.

\section{La formation des noms en -iste et en -ista}

Les noms dits d'agent font l'objet de nombreuses études. En termes de complexité formelle, on trouve en français et en italien des lexèmes construits par dérivation, par composition morphologique ou syntaxique. D'un point de vue sémantico-référentiel, les noms dits d'agents sont corrélables à des activités, des habitudes, des traits de caractère, des situations sociales, des ethnies, des lieux, des idéologies, des convictions, des hiérarchies, des productions intellectuelles ...

Souvent, chacune de ces classifications ne prend en compte l'autre que partiellement. En effet, dans les travaux de sémantique et de lexicologie, on examine les noms d'agents exclusivement du point de vue de leur classification sémantique (sans prétendre à l'exhaustivité, citons (Anscombre, 2001, 2003 ; Busa, 1997 ; Cruse, 1973 ; Flaux \& Van de Velde, 2000; Gross, 1994, 2011)), sans corréler les résultats obtenus à leur complexité morphologique ou à leur construction syntaxique. À l'inverse, en morphologie, les données analysées sont examinées à la lueur du modèle morphologique qu'elles ont en commun. Quand l'étude s'intéresse au type sémantique de ces données, c'est sans prendre la peine de rapprocher celles-ci d'autres noms, construits autrement, mais relevant de la même classe sémantique. En d'autres 
termes, les noms d'agents sont examinés sous l'angle onomasiologique ou sémasiologique, et le croisement des deux points de vue n'est que rarement envisagé.

Roché (2011a) et Lo Duca (2004), chacun à leur manière, sont conscients de ce problème, comme le montre la monographie qu'ils consacrent, respectivement, à -iste (section 3.1) et -ista (section 3.2). En particulier, Roché (2011b : 20-22) souligne la difficulté qu'il y a à réaliser l'étude morphologique d'une série de données de manière à privilégier les processus de nomination. En effet, l'auteur remarque, à juste titre, qu'un schème de nomination n'a pas nécessairement de traduction sémasiologique attitrée : par exemple, le français ne dispose pas d'un procédé morphologique destiné à former les singulatifs (individu appartenant à un groupe). Et, à l'inverse, un procédé constructionnel se spécialise rarement dans la construction de formes appartenant à une classe sémantique particulière.

Dans notre étude, nous tentons de recouper ces deux questions, illustrées par la suffixation en -iste et -ista, prise sous l'angle de la comparaison du français avec l'italien. Notre réflexion comporte deux versants : (a) le déséquilibre entre processus de nomination et procédé constructionnel, et (b) le français et l'italien vus comme la traduction l'un de l'autre :

(a) Le fait que les approches onomasiologique et sémasiologique ne coïncident pas s'observe en effet avec les suffixations en -iste et -ista, qui nous intéressent ici. Dans ces deux langues, un nom construit au moyen de ce suffixe est réputé désigner soit l'adepte d'une idéologie, soit l'acteur d'une activité. Le nom d'activité ou d'idéologie correspondant est souvent (mais pas toujours) construit en Xisme (resp. Xisma) et donc indirectement relié à Xiste (resp. Xista). Le procédé de construction ne forme donc pas un seul et unique type sémantique de noms. Et, inversement, les noms d'acteurs, tout comme les noms d'adeptes, ne sont pas exclusivement le produit de la dérivation en -iste ou -ista.

(b) La suffixation en -iste est souvent décrite comme homogène à travers les langues européennes, qui pour la plupart possèdent un suffixe cognat, induisant ainsi une équivalence de traduction potentiellement régulière. C'est ce que nous tentons de vérifier, en examinant les noms suffixés en -ista en italien et les noms suffixés en -iste en français.

\subsection{Le français}

L'une des études récentes les plus exhaustives en français sur les dérivés en Xiste est celle de Roché (2011a), qui traite des variétés sémantiques de Xisme (exorcisme, communisme, parachutisme, parallélisme). L'hypothèse défendue est que la dérivation en -isme se définit comme une règle mettant en jeu trois modèles, dont deux sont apparentés à des noms en -iste. Le premier, qui est le mieux représenté dans le lexique, regroupe les noms que nous qualifierons d'idéologies (communisme) et de leurs adeptes (communiste), et le second concerne les noms d'activités (parachutisme) et des agents qui les pratiquent (parachutiste). De manière plus anecdotique, l'auteur relève des Xiste, étrangers aux modèles majoritaires, et pouvant s'interpréter comme singulatifs (chøur $>$ choriste) ou ethniques (Brive $>$ briviste).

La présentation de ces deux modèles s'attache en particulier à décrire et analyser les situations lacunaires, c'est-à-dire les paires Xisme/Xiste où l'un des deux membres n'est pas attesté, ainsi que les cas de concurrence entre Xiste et d'autres noms d'agent ou d'adepte.

Dans le premier modèle, l'absence des Xiste s'explique le plus souvent au moyen du principe général d'économie : la langue réinvestit la forme d'un dérivé existant, en lui conférant un autre emploi, au lieu de créer une forme nouvelle. La place qu'aurait dû occuper Xiste est déjà prise par un autre nom, de la même famille dérivationnelle que Xisme. Le sens de ce remplaçant acquiert donc une valeur axiologique dans l'un de ses emplois: c'est ainsi que conservateur désigne non seulement une fonction professionnelle, mais également le partisan du conservatisme, et que préraphaélite dénote le tenant du mouvement esthétique du préraphaélisme. La plupart du temps, Xiste est attesté (conservatiste, préraphaéliste) mais beaucoup moins employé que son concurrent polysémique. 
Quand le nom en -isme est préfixé par anti-, comme anticléricalisme et antiparlementarisme, qui désignent, respectivement, le mouvement auquel adhèrent ceux qui sont anticléricaux ou antiparlementaires, alors le nom de celui qui défend cette idéologie résulte de la nominalisation de l'adjectif de relation (un antiparlementaire, un anticlérical). Le doublon en -iste est utilisé beaucoup plus rarement.

Enfin, quand Xisme n'est plus/pas motivé, c'est-à-dire quand $X$ est ininterprétable en synchronie, alors Xiste s'emploie beaucoup moins que le convert désadjectival. C'est le cas notamment des constructions sur protestant, nazi, chiite, cathare, sceptique, cynique, bolchevik.

Dans le second modèle, les cas de concurrence à Xiste sont plus variés. M. Roché distingue les couples Xisme/Xiste dérivés d'un verbe (exorciser > exorcisme, exorciste), les Xiste dérivés d'un nom d'activité sans Xisme associé (grève > gréviste), les noms en Xisme dérivés de nom d'agent, donc dépourvus de Xiste dans leur famille morphologique (cannibale $>$ cannibalisme, nomade $>$ nomadisme, proxénète $>$ proxénétisme), et les paires Xisme/Xiste dérivés d'un $X$ nominal. Dans ce dernier cas, $X$ renvoie au procès (parachute $>$ parachutisme, parachutiste) ou désigne un participant au procès autre que l'agent (justement réalisé par Xiste) : l'objet dont s'occupe Xiste (auberge $>$ aubergiste, fleur $>$ fleuriste) ou son instrument (violon > violoniste), ou le lieu scénique (Alpes $>$ alpiniste). Le nom d'activité Xisme y est souvent lacunaire.

Les noms en -iste renvoient exclusivement à des humains, ce qui différencie ce mode de formation de la suffixation en -eur, par exemple, qui construit certes des noms d'agent humains, mais aussi des noms d'instruments. C'est pourquoi les noms en -isme dénotant des activités non humaines sont dépourvues de parent en -iste (cataclysme, volcanisme). Il en va de même pour les noms d'activité non agentive, mais affectant un individu (zozotisme, alcoolisme, nicotinisme, saturnisme). D'autres lacunes de -iste sont certainement dues à leur évincement au profit d'un affixe plus populaire. C'est le cas de hypnotisme/hypnotiseur.

Quand il désigne l'agent de l'activité Xisme dans laquelle la base $X$ désigne un autre participant, Xiste, resté longtemps cantonné à la sphère dite savante (évangéliste, juriste), entre en concurrence directe avec les formes en Xeur et Xier. L'émergence de Xiste, doublonnant ses concurrents ou se substituant à eux, est facilitée par l'un des facteurs :

- $\quad$ phonologiques, qui favorisent aubergiste plutôt que *aubergier ; fumiste plutôt que *fuméier

- $\quad$ sociolinguistiques, qui exploitent le prestige résiduel dont dispose -iste comme formateur de noms «savants» (métiers intellectuels, fonctions religieuses). Ce prestige explique l'émergence de harpiste, qui remplace harpier, aux côtés de cymbalier, soulignant ainsi la noblesse du premier instrument par rapport au second.

Enfin, le résultat des deux facteurs ci-dessus est magnifié par un effet de série, qui conduit à la production de nouveaux Xiste conçus uniquement par proximité référentielle avec ceux qui sont générés pour l'une ou l'autre des 2 raisons ci-dessus.

En résumé, l'absence d'un dérivé en Xiste peut être causée par différents facteurs :

- De nature sémantique : aucun agent n'est prévisible en lien avec Xisme, ou bien le nom apparenté à Xisme désigne un humain dont le rôle est non agentif;

- De nature phonologique : un autre affixe (souvent -eur ou -ier) est habituellement mieux implanté pour exprimer ce que doit exprimer Xiste;

- De nature lexicale (que M. Roché appelle 'recyclage') : le fruit de la nominalisation de n'importe quel type morphologique d'adjectif peut s'observer à la place de Xiste quand Xisme renvoie à une idéologie ;

- De nature morphologique : les noms d'idéologie en anti-, ainsi que ceux dont la base $(X)$ est non motivée, sont très peu souvent apparentés à un nom en -iste. Les noms Xisme dérivés de noms d'activité sont privés de Xiste. 
D'après cette étude, il est normal de s'attendre à ce que les Xiste 'adepte' soient plus nombreux que les Xiste 'acteurs', si on s'en tient au déséquilibre quantitatif des modèles, mais, en même temps, à ce que les Xiste 'adeptes' soient des dénotations minoritaires, concurrencées par d'autres formes (désadjectivales), et que les Xiste 'acteurs' soient les doublures de noms en -eur ou en -ier, auxquels ils se substituent parfois.

\subsection{L'italien}

L'ouvrage de référence en morphologie italienne, « La Formazione delle parole in italiano » (Grossman et Rainer, 2004) décrit la suffixation en -ista principalement dans le chapitre consacré aux noms d'agent (Lo Duca, 2004 :191-215). Ce chapitre adopte une classification relativement similaire à celle proposée pour le français dans (Roché, 2011a), même si la formation de Xista y est traitée de manière indépendante de celle des noms en -ismo. M.G. Lo Duca adopte une démarche plus onomasiologique, puisque les noms de la forme Xista sont classés parmi les noms d'agent dénominaux, au même titre que d'autres dérivés suffixaux : Xaio (fioraio - Fr : fleuriste ), Xaro (campanaro - Fr : carillonneur), Xaiolo, Xaiula, Xaialo (fruttaiolo - Fr : marchand de fruit), Xiere, Xiero (barbiere - Fr : coiffeur), Xario (bibliotecario - Fr : bibliothécaire), Xino (postino - Fr: facteur), Xante (aiutante - Fr: aide), Xiano (darviniano - Fr: darwinien).

Dans la famille sémantique des noms agentifs, Xista est prédominant dans l'expression des noms de métier. La raison invoquée par M.G. Lo Duca (comme celle que propose M. Roché pour le français) est que la suffixation en -ista dispose d'une sorte de prestige, acquis à l'origine par l'emploi de ce suffixe dans la formation de noms savants, et dont témoigne l'existence de modèles de suffixation quasiidentiques dans les autres langues européennes (notamment en français avec -iste et en anglais avec -ist).

M.G. Lo Duca remarque la grande adaptabilité de la suffixation en -ista, qui se manifeste dans l'absence de contrainte portant sur la structure, la catégorie ou la nature sémantique des bases sélectionnées. Cellesci peuvent être des noms propres (calvinista $<$ Calvin, ferrarista $<$ Ferrari) ou des noms communs ; ces noms relèvent d'une large gamme de types sémantiques : humains (femminista $<$ femmina $-\mathrm{Fr}$ : femme) ou non,(stragista $<$ strage - Fr: tuerie), comptables (fumettista $<$ fumetto - Fr : bande-dessinée) ou massifs (fuochista < fuocho - Fr: feu), concrets (pianista < pianoforte - Fr: piano) ou abstraits, $\left(\right.$ chiusurista $<$ chiusura $-\mathrm{Fr}$ : fermeture) ${ }^{1}$. Le nom de base peut être simple (flautista $<$ flauto $-\mathrm{Fr}$ : flutte) ou morphologiquement construit (decisionista < decisione - Fr: décision, giustificazionista $<$ giustificazone - Fr : justification), et sa structure prosodique est non contrainte en termes de nombre de syllabes. Si elle est souvent nominale, la catégorie de base de la suffixation en -ista peut être également adjectivale (legittimista < legittimo - Fr : légitime, qualunquista < qualunque - Fr : quelconque), verbale (apprendista < apprendere - Fr: apprendre), adverbiale (dietrista $<$ dietro $-\mathrm{Fr}$ : derrière) ; il peut s'agir d'un composé ou d'une locution (duecentometrista $<$ duecento metri - Fr : deux cents mètres), d'un emprunt (belletrista $<$ Fr : Belles-Lettres), d'un acronyme (tirrista $<$ TIR) ou d'un sigle (keghebista $<\mathrm{KGB})$.

Tout comme les noms en -iste du français, les Xista désignent des individus qui participent à une activité, sont spécialistes d'une discipline, adhèrent à une idée, ou se caractérisent par un comportement. M.G. Lo Duca propose donc de répartir ces noms en deux classes :

- les noms d'agents dits 'classifiants', qui sont acteurs d'une activité professionnelle. Le groupe que forment ces noms comprend : les noms de joueurs d'instrument de musique (flautista $<$ flauto $-\mathrm{Fr}$ : flutte), de rédacteurs professionnels (cronista $<$ cronaca - Fr: chronique), de spécialistes de disciplines littéraires ou scientifiques (latinista < latino - Fr: latin), les noms qui désignent des artistes peintres (rittratista $<$ rittrato $-\mathrm{Fr}$ : portrait, acquarellista $<$ acquarella - Fr : acquarelle), des sportifs, et des conducteurs de véhicule (aliscafista < aliscafo - Fr: hydroglisseur, autociternista $<$ autociterna - Fr : camion-citerne) ;

- les noms d'agent dits 'caractérisants', qui peuvent dénoter: les personnes qui adhèrent à une idéologie (zappatista<Zappata), ou à un mouvement d'opinion (abortista < aborto - Fr: avortement), les membres d'un groupe ou d'une association (camorrista < camorra - Fr : mafia 
napolitaine), les individus que caractérisent une tendance (consumista $<$ consumo $-\mathrm{Fr}$ : consommation) ou un trait de caractère (pessimista - Fr : pessimiste).

Comme le fait remarquer M. Roché pour le français, les noms en -ista peuvent être formés sur des noms d'activité, et donc ne pas être appariés à un nom en -ismo. C'est ainsi qu'à côté de quelques couples comme giornalista / giornalismo (Fr : journalisme), ou nutrizionista ( $\mathrm{Fr}$ : nutritionniste) / nutrizionismo, on trouve beaucoup plus souvent des noms d'agent classifiants en -ista sans relation avec un nom en -ismo : pianista (Fr: pianiste), camionista (Fr : camionneur), gallerista (Fr : galleriste).

Toujours à l'image de ce que M. Roché remarque pour le français, -ista peut être concurrencé par d'autres suffixes dans la formation de noms d'agents d'activité, à partir de la même base nominale; le suffixe employé est le reflet de la hiérarchie dans laquelle sont placées les activités correspondantes dans l'esprit des locuteurs : c'est ainsi que le giornalista, qui rédige les articles d'un journal se distingue du giornalaio qui vend les journaux.

Les appariements entre Xista et Xismo sont en revanche (en italien comme en français) plus réguliers lorsque Xista est qualifiable de nom d'agent caractérisant, et plus particulièrement lorsqu'il dénote l'adepte ou le partisan qui soutient une idéologie ou une opinion : peronista/peronismo (Fr: péronisme), fascista/fascismo ( $\mathrm{Fr}$ : fascisme).

\subsection{Bilan}

En substance, les études monolingues que nous venons de résumer dans cette section considèrent les noms en -iste et -ista suivant deux perspectives distinctes : la monographie de M. Roché étudie Xiste suivant son rapport morphologique avec Xisme, et émet l'hypothèse que la formation des noms en -iste, tout comme celle des noms en -isme, obéit à deux modèles relevant d'une même règle ; quant à l'article de M.G. Lo Duca, il s'intéresse à Xista en tant qu'output d'une règle de construction de noms d'agent, comparée aux autres modes de formation produisant le même type sémantique de noms. Tout en ayant des objectifs différents, des aprioris théoriques distincts, et des approches complémentaires, les deux articles postulent des hypothèses comparables et parviennent à des résultats similaires : pour les deux auteurs, -ista et -iste se retrouvent dans plusieurs langues européennes, forment principalement des noms d'adeptes qui soutiennent une idéologie (réalisée souvent en -isme ou -ismo), ou des noms d'agents d'activité. À l'origine, les activités exercées par ces agents étaient assimilables à des métiers savants ou prestigieux. Dans les deux langues, on signale que le nom d'agent peut exister sans son parent en -isme ou -ismo, et que, à l'inverse, il peut être concurrencé, voire évincé, par un autre nom d'agent formé sur la même base.

À première vue, -ista et -iste semblent être les deux faces, l'une italienne, l'autre française, d'un même mode de formation, dont les différences de comportement ne seraient imputables qu'aux contraintes phonologiques de chaque langue. Si cette hypothèse est vraie, alors elle doit se refléter dans un dictionnaire bilingue, par un mécanisme de traduction $\mathrm{It} \rightarrow \mathrm{Fr}$ et $\mathrm{Fr} \rightarrow$ It symétrique à tous les points de vue : même nombre de Xiste et Xista sources et cibles, même proportion de noms d'agents classifiants versus caractérisants dans les deux langues, etc. Il faut cependant prendre en compte un dernier paramètre : celui de la plasticité de -ista évoquée par M.G. Lo Duca, et conduisant ce suffixe à pouvoir sélectionner en position de base des formes construites par la syntaxe : duecentometrista $<$ duecento metri (Fr: 'coureur du 200 mètres'), saccopelista < sacco a pelo ('Fr: 'qui dort dans un sac de couchage').

Dans les sections qui suivent, nous examinons les équivalents de traduction pour ces formes proposées par un dictionnaire bilingue, et nous étudions dans quelle mesure la symétrie dans la construction de Xiste et Xista est totale ou partielle. 


\section{La juxtaposition des données}

Dans cette étude, nous avons donc utilisé comme source de données le dictionnaire italien-français et français-italien Garzanti sur CD-Rom (2006), qui contient 65308 entrées dans le sens It $\rightarrow$ Fr et 62046 entrées dans le sens $\mathrm{Fr} \rightarrow$ It. Pour chaque sens de traduction, nous avons tout d'abord extrait toutes les entrées contenant comme mot vedette un lexème construit en -iste (ou -ista, suivant la langue source). Nous avons ensuite manuellement procédé à une classification de ces lexèmes, en nous fondant sur les résultats proposés dans les études monolingues résumées à la section 3. Ainsi, nous avons distingué deux classes. La première est celle des agents caractérisants, et reprend le modèle 2 proposé par M. Roché et regroupe les adeptes et les comportements. La seconde, comporte les agents classifiants, correspond au modèle 1 de M. Roché, et regroupe les noms d'acteurs et de spécialistes.

Quand une entrée est polysémique, et dispose d'équivalents de traduction distincts, nous en avons fait deux entrées distinctes, chacune avec sa propre traduction: ce choix reflète l'adoption de l'hypothèse linguistique postulée par Fradin \& Kerleroux (2003) qui considèrent qu'un lexème est sémantiquement spécifié. Si une forme est ambiguë, alors elle est correspond à plusieurs lexèmes. Par exemple, nous avons levé l'ambiguitté portée par l'italien cubista en doublant le nombre d'entrées : cette forme désigne en effet soit un cubiste, i.e. un adepte du cubisme, soit un professionnel de la danse en boîte de nuit (que Garzanti traduit par gogo-danseur).

Une fois cette classification établie, nous nous sommes intéressés aux traductions. Certaines entrées proposaient plusieurs traductions synonymes (parfois jusqu'à 4). Nous avons considéré toutes les traductions de manière équivalente, bien qu'il soit d'usage en lexicographie bilingue de classer les équivalents selon un ordre de préférence (ordre, d'ailleurs, rarement explicité par les éditeurs de dictionnaires). En outre, il faut souligner ici que nous avons centré notre analyse sur les lexèmes construits (output des règles), sans évaluer l'équivalence sémantique des bases (les inputs). À ce titre, la notation utilisée pour des paires de mots construits proposés par le dictionnaire (p. ex. : Xista $\rightarrow$ Xiste, ou $X i s t a \rightarrow$ Xeur) ne présuppose en rien le degré d'équivalence entre les bases française et italienne (les ' $\mathrm{X}$ ') Ainsi, nous considérons de la même manière des équivalents tel que scooterista $\rightarrow$ scootériste et vetrinista $\rightarrow$ étalagiste, même si l'équivalence sémantique entre les deux bases diffère selon les cas: l'italien vetrina ( $\mathrm{Fr}$ : vitrine) et le français étalage ont un degré d'équivalence moindre que les deux bases du premier exemple (scooter). Une étude plus étendue mériterait évidemment de se pencher sur ces différents niveaux d'équivalence (de règle, d'input et d'output).

Nous avons ensuite réparti les équivalents de traduction suivant différents critères. En particulier, nous avons distingué les traductions réalisées par un mot non construit ou par un emprunt à une langue tierce, de celles formées dans la langue cible par suffixation, par préfixation, composition morphologique ou syntaxique. Enfin, nous avons relevé, dans de rares cas, une définition sous forme de paraphrase en guise d'unique traduction (abortista $\rightarrow$ qui est favorable à l'avortement).

La section 5 ci-dessous décrit la troisième étape de l'analyse contrastive, i.e. la comparaison proprement dite. Les résultats de la classification sémantique des constructions en -ista et en -iste sont analysés dans la section 5.1 ; ensuite, nous examinons plus en détail les équivalents de traduction en fonction de leur mode de formation : nous nous interrogeons en particulier sur les formes qui ne sont pas construites en -iste (section 5.2). La section 6 se consacre ensuite exclusivement aux différents procédés français présents parmi les équivalents des lexèmes italiens en -ista.

\section{La comparaison des données}

\subsection{Répartition de Xista et Xiste par type sémantique}

Le tableau 1 présente la répartition des différents types sémantiques des dérivés en Xiste et en Xista dans les deux langues sources. Cette première mesure de comparaison des deux langues sources est révélatrice de deux points essentiels. 
Premièrement, la partie It $\rightarrow$ Fr comporte 262 entrées de plus (soit env. $45 \%$ ) pour un dérivé en Xista que d'entrées en Xiste dans la partie Fr $\rightarrow$ It. Cet écart, même s'il peut être influencé par certains choix éditoriaux des rédacteurs des deux sections du dictionnaire, semble montrer que nous avons à faire à une importante divergence entre Xista et Xiste, malgré leur apparente similitude formelle et sémantique.

Deuxièmement, nous constatons que, parmi les dérivés, la proportion de chaque valeur sémantique est sensiblement identique dans les deux langues : nous obtenons environ $40 \%$ de noms d'agents classifiants, et environs $60 \%$ de noms d'agents caractérisants.

\begin{tabular}{|l|c|c|c|c|}
\hline & \multicolumn{2}{|c|}{ Xiste $(\mathbf{F r} \rightarrow \mathbf{I t})$} & \multicolumn{2}{c|}{ Xista $(\mathbf{I t} \rightarrow \mathbf{F r})$} \\
\hline Type sémantique & nbr. & $\mathbf{\%}$ & nbr. & $\mathbf{\%}$ \\
\hline $\begin{array}{l}\text { Agents caractérisants } \\
\text { (adepte ou comportement) }\end{array}$ & 353 & 61,3 & 509 & 60,7 \\
\hline $\begin{array}{l}\text { Agents classifiants } \\
\text { (acteur ou spécialiste) }\end{array}$ & 223 & 38,7 & 329 & 39,3 \\
\hline Total & $\mathbf{5 7 6}$ & & $\mathbf{8 3 8}$ & \\
\hline
\end{tabular}

Tableau 1 : Répartition des valeurs sémantiques des dérivés en Xiste/Xista

\subsection{La traduction de Xiste et Xista}

Nous regroupons ensuite les traductions proposées par le Garzanti pour ces noms suffixés (Xista ou Xiste), en fonction de leur classe sémantique et nous calculons la proportion d'entrées qui présentent au moins une traduction construite avec le suffixe cognat. Ces proportions sont présentées dans le tableau 2. Rappelons ici que les proportions sont calculées sur l'ensemble des traductions proposées (une entrée pouvant contenir plusieurs propositions de traduction).

\begin{tabular}{|l|c|c|c|c|c|c|}
\hline & \multicolumn{3}{|c|}{ Fr $\rightarrow$ It } & \multicolumn{3}{c|}{ It $\rightarrow$ Fr } \\
\hline Type sémantique & entrées & Xiste $\rightarrow$ Xista & $\%$ & Entrées & Xista $\rightarrow$ Xiste & $\%$ \\
\hline Agents caractérisants & 353 & 242 & $68.6 \%$ & 509 & 266 & $52,3 \%$ \\
\hline Agents classifiants & 223 & 198 & $88.8 \%$ & 329 & 282 & $85,7 \%$ \\
\hline Total & $\mathbf{5 7 6}$ & $\mathbf{4 4 0}$ & $\mathbf{7 6 . 4 \%}$ & $\mathbf{8 3 8}$ & $\mathbf{5 4 8}$ & $\mathbf{6 5 . 4 \%}$ \\
\hline
\end{tabular}

Tableau 2 : Proportion des dérivés en Xiste/Xista ayant au moins une traduction construite avec le suffixe cognat

Les pourcentages affichés dans le tableau 2 appellent plusieurs commentaires. Globalement, il y a plus de noms en -iste traduit en Xista (76.4\%) que de noms en -ista traduit en Xiste $(65,4 \%)$, ce qui semble montrer, par un effet de miroir, une plus grande disponibilité du modèle Xista, par rapport à Xiste. Même si cette divergence varie selon les valeurs sémantiques du suffixe (cf. ci-dessous), elle laisse entrevoir une absence de symétrie entre ces deux formations, malgré leur parenté formelle apparente.

Si les noms d'agents caractérisants possèdent un équivalent de traduction majoritairement construit au moyen du suffixe cognat (entre $85 \%$ et $88 \%$ des cas), la situation est tout autre pour la formation des noms d'agents classifiants (acteurs ou spécialistes). La proportion de Xiste équivalents de traduction de Xista est très inférieure $(52,3 \%)$ à celle des noms d'agents traduits du français Xiste, et construits au 
moyen du suffixe cognat en italien $(68,6 \%)$. On voit ainsi que l'italien construit très facilement des noms d'agents classifiants en -ista, là où le français semble plus rétif à le faire.

Si le parallélisme des proportions pour les différentes valeurs sémantiques confirme la similarité du fonctionnement dans la formation en Xiste et en Xista (cf. tableau 1), le détail des chiffres présentés au tableau 2 montre qu'un nombre important de lexèmes n'ont pas d'équivalent formé au moyen du suffixe cognat dans le sens It $\rightarrow$ Fr, principalement en ce qui concerne les noms d'agents classifiants.

Le reste de l'article s'intéresse donc aux paires de traduction It $\rightarrow \mathrm{Fr}$, et particulièrement au cas où les Xista ne sont pas traduits par un nom construit en -iste.

\section{L'éclairage de l'analyse contrastive sur le français}

Dans la section précédente, nous avons présenté la comparaison des deux langues à partir des données extraites du dictionnaire bilingue, en soulignant les écarts entre l'italien et le français. Dans ce qui suit, nous mettons particulièrement l'accent sur le français, en analysant les différents éléments utilisés pour traduire en français les lexèmes italiens en Xista. Le prisme ainsi utilisé permet d'adopter une approche onomasiologique pour étudier les noms d'agents en français.

\subsection{Données et statistiques}

Dans le dictionnaire bilingue utilisé, chaque entrée propose une ou plusieurs possibilités de traduction. Ce chiffre, bien que hautement influencé par d'autres facteurs (choix éditoriaux, contraintes de place), peut être perçu comme un témoin de l'hésitation à laquelle conduit la traduction de certains lexèmes en Xista. Mais il sert surtout d'indicateur à la grande variété formelle des noms d'agents en français, qu'ils désignent des adeptes ou des acteurs d'activité. C'est par le biais de cette offre en matière de traductions que peuvent s'articuler les points de vue sémasiologique et onomasiologique de la dérivation en -iste.

Le tableau 3 présente le pourcentage d'entrées en italien proposant 1, 2 ou 3 traductions différentes, en fonction de la valeur sémantique du lexème vedette. Les agents classifiants ont plus rarement une seule possibilité de traduction que les agents caractérisants.

\begin{tabular}{|c|c|c|c|}
\hline & $\mathbf{1}$ & $\mathbf{2}$ & $\mathbf{3}$ \\
\hline agents caractérisants & $92.64 \%$ & $7.36 \%$ & $0.00 \%$ \\
\hline agents classifiants & $80.85 \%$ & $18.18 \%$ & $0.97 \%$ \\
\hline
\end{tabular}

Tableau 3 : Nombre de traduction par type sémantique

Comme expliqué dans la section 4, nous avons procédé à une classification de chaque traduction, qu'elle soit morphologiquement construite (en spécifiant le procédé) ou non. La répartition des différents types de traduction en fonction du type sémantique est également très informative. Le Tableau 4 présente les proportions calculées pour les deux types sémantiques d'agents. Les premières colonnes donnent le pourcentage de traductions construites au moyen des trois suffixes les plus fréquents (-iste, -eur, -ier), suivi d'un ensemble «autre moyen morphologique » qui regroupe les autres procédés de dérivation (affixation - notamment en -ant, et composition morphologique). La colonne «composé syntaxique » regroupe les traductions réalisées essentiellement sous la forme « $\mathrm{N}$ prep $\mathrm{N}$ », faisant intervenir les prépositions telle que $d e$, $\grave{a}$, ou en. La colonne "définition» regroupe les traductions exprimées sous forme de définition. Enfin, la dernière colonne concerne les traductions qui sont soit des lexèmes morphologiquement non construits (NC), soit des emprunts, i.e. des mots n'appartenant pas au fonds lexical du français. 


\begin{tabular}{|l|c|c|c|c|c|c|c|}
\hline en $\%$ & -iste & -eur & -ier & $\begin{array}{c}\text { autre moyen } \\
\text { morpho. }\end{array}$ & $\begin{array}{c}\text { composé } \\
\text { syntaxique }\end{array}$ & définition & emprunt/NC \\
\hline caractérisant & $80.8 \%$ & $0.9 \%$ & $0.3 \%$ & $4.3 \%$ & $6.6 \%$ & $4.0 \%$ & $3.2 \%$ \\
\hline classifiant & $43.5 \%$ & $14.4 \%$ & $4.9 \%$ & $12.4 \%$ & $19.5 \%$ & $1.1 \%$ & $4.1 \%$ \\
\hline
\end{tabular}

Tableau 4 : type de traduction en fonction du type sémantique

Ces chiffres appellent plusieurs commentaires d'ordre général. Comme le laissaient prévoir les estimations précédentes, les noms d'agents caractérisants sont le plus souvent traduits par une construction en Xiste. En revanche, les noms en Xista désignant des agents classifiants donnent lieu à une plus grande variété de traductions, aux côtés de Xiste. Ces traductions peuvent être des dérivés, suffixés en -eur, -ier ou -ant (qui, suivant M. Roché, sont des concurrents naturels et directs de -iste), soit des composés syntaxiques.

\subsection{Description qualitative}

\section{Les noms d'agents caractérisants}

Les noms d'agents caractérisants, et, parmi eux, les noms d'adeptes de la forme Xista, sont, comme le montre le tableau 4, traduits à $80 \%$ par un nom suffixé de la forme Xiste. Certes, d'autres modes de construction morphologique concurrencent en français la traduction Xiste de Xista. Conformément à l'analyse proposée dans (Roché, 2011a) à la section 3.1, il s'agit le plus souvent de cas de recyclage d'adjectifs substantivés en -ien $:$ sansimonista $\rightarrow$ saint-simonien, stalinista $\rightarrow$ stalinien.

Cependant, il est intéressant de noter que la traduction de Xista peut être réalisée sous la forme d'un composé «N1 prep N2 » où N1 appartient au domaine conceptuel des noms d'adeptes et N2 est une traduction de X : classicista $\rightarrow$ partisan du classicisme, neoclassicista $\rightarrow$ disciple de l'art néoclassique.

\section{Les noms d'agents classifiants}

Quand ils constituent la traduction d'une entrée en Xista, et hormis les cas de traduction en -iste, les noms d'agents d'activité sont principalement construits au moyen des affixes dont M. Roché fait mention, à savoir -eur dans $14.4 \%$ des cas, (e.g. hockeista $\rightarrow$ hockeyeur, piastrellista $\rightarrow$ carreleur), et -ier dans $4.9 \%$ des cas, (e.g. cementista $\rightarrow$ cimentier, gruista $\rightarrow$ grutier). C'est également uniquement pour cette valeur sémantique que l'on trouve l'emploi d'emprunts, surtout de l'anglais (barista $\rightarrow$ barman, microfonista $\rightarrow$ perchman). Enfin, parmi ces agents classifiants, le sous-groupe des « spécialistes » présente un nombre important de formations en -ant (ebraista $\rightarrow$ hébraïsant, arabista $\rightarrow$ arabisant).

Parmi les autres moyens morphologiques utilisant la dérivation, notons notamment la suffixation en -ien, exceptionnellement dénominale (farmacista $\rightarrow$ pharmacien), (cf. (Lignon \& Plénat, 2009) pour une analyse de ce cas d' «échangisme suffixal»), et les nominalisations d'adjectifs en -aire, (azionista $\rightarrow$ actionaire)

De nombreuses traductions de Xista font appel aux procédés de composition. Nous distinguons ici composition morphologique et composition syntaxique : en cela, nous nous conformons aux critères proposés à l'origine par Corbin (2000). En conséquence, nous rangeons la traduction de Xista dans la classe des composés morphologiques, quand elle ne comporte que des constituants appartenant à des catégories majeures ; à l'inverse, nous la plaçons parmi les composés syntaxiques, quand elle met en jeu au moins un grammème : préposition ou déterminant.

Les composés morphologiques relevés parmi les traductions de Xista sont tous formés de deux noms (pour un panorama complet de la composition en français, voir (Fradin, 2009)). Quelques-uns sont qualifiables de composés standards, ou ordinaires (fotocronista $\rightarrow$ reporteur-photographe). Les autres sont des composés dits savants ou néoclassiques (cf. Dal \& Amiot, 2008 ; Namer \& Villoing, 2007) : 1'un 
au moins de leurs constituants est un radical issu du latin et du grec, et le constituant sémantiquement recteur est placé à droite (dirigibilista $\rightarrow$ aéronaute, necrologista $\rightarrow$ nécrologue, callista $\rightarrow$ pédicure).

La composition syntaxique, le plus souvent de la forme « N1 prep N2», intervient dans $20 \%$ environ des traductions de noms en Xista d'agents classifiants. Dans de nombreux cas, N1 est un supertype de Xista et $\mathrm{N} 2$ est soit la traduction de X (trattorista $\rightarrow$ conducteur de tracteur, rugbista $\rightarrow$ joueur de rugby), soit sémantiquement relié à X (ambientalista $\rightarrow$ peintre d'intérieur). Notons également que cette option de traduction relève parfois d'un choix éditorial. Il est apparu en effet que parfois, l'équivalent construit en français existait, mais que le dictionnaire s'était contenté d'une traduction sous forme de composé. Dans certains cas, ce choix est conforme à la fréquence avec laquelle cette construction est employée, et qui dépasse dans l'usage la fréquence d'emploi de Xiste.

Mais dans d'autres cas, le choix du dictionnaire de traduire Xista par un composé n'est pas justifié par la fréquence d'emploi : le composé s'utilise en effet moins souvent que Xiste : par exemple, l'interrogation de Google avec sauteur à la perche, traduction fournie par Garzanti de astista, ramène environ 50,000 pages, alors que la même requête, formulée avec perchiste, renvoie 163,000 résultats.

\subsection{Bilan}

L'analyse contrastive des formations en Xiste en français avec celles en Xista en italien a montré une hétérogénéité dans les constructions prises comme traductions les unes des autres : les formes en Xista de l'italien ne donnent pas toujours lieu à des formes en Xiste en français. Mais l'analyse chiffrée des valeurs sémantiques des noms en -iste, que nous avons présentée à la section 5 montre que ce déséquilibre constructionnel dépend de la classe sémantique de Xista: la structure morphologique des noms d'agents caractérisants est stable en traduction (Xista se traduit quasi-uniformément en Xiste), alors que celle des noms d'agents classifiants est généralement imprédictible (moins de la moitié des traductions de Xista sont en Xiste).

Ce décalage provient de certaines caractéristiques en français. En effet, la formation en Xiste semble moins naturelle quand le nom produit désigne un acteur. Roché explique partiellement ce fait par l'utilisation possible d'autres suffixes concurrents, comme -eur, -ier ou -ant, ce que notre étude a par ailleurs confirmé empiriquement. Cette étude a également permis de montrer que des modes de constructions morphologiques peuvent entrer en concurrence avec des patrons syntaxiques de structure «N1 prep N2 », donc formellement réguliers, et qui impliquent également le même type de relation sémantique que celle observable dans les cas de composition morphologique (comme nous l'avons vu avec « N1 prep N2 », où N1 est le super-type, souvent construit, de Xista, et N2 l'équivalent de traduction de $\mathrm{X})$.

Enfin, l'approche contrastive permet également d'expliquer les non-réalisations en Xiste en français par la sur-représentativité de certaines classes sémantiques de noms en -ista de italien. Par exemple, parmi les Xista agents classifiants traduits par un composé syntaxique, on remarque un nombre important de noms de sportifs (bobbista $\rightarrow$ coureur de bobsleigh, de bob, aliantista $\rightarrow$ pilote de planeur, giavellottista $\rightarrow$ lanceur de javelot). Un autre groupe saillant est celui des conducteurs d'engin mécanique. Vraisemblablement, le modèle auto $\rightarrow$ autista a inspiré un grand nombre de dérivés en italien appartenant au même type conceptuel, sans équivalent morphologiquement construit en français. C'est le cas de elicotterista $\rightarrow$ pilote d'hélicoptère, furgonista $\rightarrow$ conducteur de fourgon, motoscafista $\rightarrow$ conducteur de canot à moteur.

Plusieurs autres domaines d'activité sont affectés par une disparité massive entre le nombre de Xista et celui de Xiste: les spécialités des artistes peintres (figurista $\rightarrow$ peintre figuratif, quadraturista $\rightarrow$ spécialiste des trompe-l'œil), ou encore le droit. Ainsi, là où l'italien semble produire sans problème des dérivés de spécialiste, le français se sert plus volontiers de paraphrases (amministrativista $\rightarrow$ spécialiste en droit administratif, matrimonialista $\rightarrow$ avocat spécialiste en droit matrimonial). 
Pour finir, remarquons que la confrontation des données de l'italien et du français souligne la grande plasticité de la suffixation en -ista comparée aux plus fortes réticences qui semblent affecter l'application de la règle en -iste. La dérivation en -ista de l'italien n'est pas sensible au type de base : elle accepte les composés (saldacontista $<$ saldaconto $(\rightarrow$ teneur de livre), centrocampista $<$ centro campo $(\rightarrow$ milieu de terrain), les composés syntaxiques (centometrista <ento metri), les expansions de collocations (correntista $<$ conto corrente $\rightarrow$ titulaire d'un compte courant, rotellista $<$ pattini a rotelle $\rightarrow$ hockeyeur sur roulettes).

Bien que réputée très peu contrainte (le français fabrique lui aussi sans peine des dérivés en -iste sur des bases de tous types catégoriels : camping-car > camping-cariste, je m'en fout $>$ je-m'en-foutiste, laisser faire > laisserfairiste, orchestre de chambre $>$ chambriste ...) la règle en -iste semble moins tolérante que son homologue en -ista: *cent-mètriste n'est pas attesté, si ce n'est sous la forme hapaxique quatrecent-métriste (seule réponse renvoyée par Google à la requête "cent-métriste », soumise en avril 2012). Le Garzanti propose d'ailleurs l'équivalent sprinter de cent mètres. Un indicateur supplémentaire de ce déséquilibre peut également s'entrapercevoir par l'examen de l'autre sens de traduction (Xiste $\rightarrow$ Xista), où seules $7,4 \%$ des traductions de Xiste sont réalisées uniquement sous la forme de constructions syntaxiques ou de paraphrases définitoires.

Finalement, le point de vue contrastif confirme l'existence de deux modèles de noms en -iste et -ista, et ne contredit pas l'hypothèse selon laquelle ces deux modèles relèvent d'une règle unique, quand on se place à un certain niveau de description (Roche, 2011a :117-sq). Mais de plus, la comparaison bilingue peut mener à une nouvelle classification des noms français d'agents, qui contribue à l'identification de nouveaux facteurs avantageant ou défavorisant les différents modes de formation, comme le laisse entrevoir ce travail sur -iste. En complétant cette étude par l'analyse contrastive inverse, il nous sera possible de formuler de manière explicite la distribution des modes de formation des noms d'agents classificants dans chaque langue, en fonction des différents paramètres mis en évidence par l'observation $\mathrm{du}$ corpus bilingue (critères morphophonologiques, prosodie, catégorie syntaxique de la base, effet de série lexicale, domaine d'activité, etc.).

\section{Conclusion et perspectives}

Dans cet article, nous avons montré l'intérêt de l'approche contrastive pour une analyse morphologique dans une approche onomasiologique.

L'étude de cas de la suffixation en -iste et en -ista a permis d'illustrer par les données qu'une approche onomasiologique pouvait amener à des conclusions différentes d'une approche sémasiologique et complémentaires à celle-ci. D'une part, les deux procédés ne forment pas un seul et même type de nom, et d'autre part, les noms d'acteurs comme les noms d'adeptes ne sont pas exclusivement le produit de la dérivation en -iste ou en -ista. L'analyse contrastive a également montré d'importantes divergences entre les procédés de l'italien et du français, pourtant décrits de manière similaire dans les études monolingues.

L’étude présentée ici a permis de confirmer de manière empirique les hypothèses formulées dans les monographies monolingues (notamment celles concernant les suffixes concurrents de -iste en français). Mais ces données ont également fait apparaître des aspects nouveaux, notamment la supériorité de la plasticité de Xista en termes de sélection (structurelle ou sémantique) des bases. Ceci est particulièrement flagrant pour la formation de noms d'agents classifiants, que le français est obligé de réaliser par d'autres moyens, morphologiques ou non.

Évidemment, les ressources utilisées pour réaliser cette étude sont sujettes à caution. Nous l'avons dit, les données dictionnairiques sont fortement influencées par des choix éditoriaux et des contraintes pratiques. Ainsi, les résultats obtenus ne constituent-ils au mieux qu'une tendance, qui nécessite d'être confirmée au moyen de corpus alignés, où les fréquences de chaque traduction pourraient être calculées. Cela permettrait de pondérer les choix de traduction opérés dans le dictionnaire.

Il n'en reste pas moins qu'une telle analyse est un bon point de départ pour une cartographie des noms d'agent. Évidemment, un élargissement est nécessaire vers d'autres suffixes agentifs (comme -ant, -eur, 
-ier). De plus, l'étude inversée (dans l'autre sens de traduction), permettrait de découvrir si l'ensemble des suffixes agentifs du français donneraient lieu à une majorité de traduction en Xista. Cet élargissement permettrait de déduire un réseau de synonymes entre les lexèmes issus de procédés constructionnels différents. Ainsi, les données contrastives permettent de rapprocher les résultats inatteignables par les approches classiques, en récoltant de manière plus exhaustive les différentes réalisations morphologiques de certaines dénominations.

Enfin, la découverte d'un important nombre de composés syntaxiques servant à désigner des agents classifiants en français (et traduisant un nom d'agent en Xista italien) soulève la question de la place de ce type de composé dans la grammaire, et plus précisément de son appartenance à la morphologie ou à la syntaxe. En d'autres termes, parce qu'elle donne accès à des moyens de dénomination autres que morphologiques, l'analyse contrastive questionne les frontières de la morphologie.

\section{Références bibliographiques}

Dictionnaire de référence

(2006) Garzanti francese : francese-italiano, italiano-francese. I grandi dizionari Garzanti. Milano, Garzanti Linguistica.

Ouvrages cités

Andor, J. (2005). A Lexical Semantic-Pragmatic Analysis of the Meaning Potentials of Amplifying Prefixes in English and Hungarian. A Corpus-based Case Study of Near Synonymy. Proceedings from The Corpus Linguistics Conference Series, 1(1).

Anscombre, J.-C. (2001). À propos des mécanismes sémantiques de formation de certains noms d'agent en français et en espagnol, Langages 143, 28-48.

Anscombre, J.-C. (2003). L'agent ne fait pas le bonheur : agentivité et aspectualité dans certains noms d'agent en espagnol et en français, Revista Complutense de Estudios Franceses 11, 11-27.

Atkins B.T.S. (2002) Bilingual dictionaries: Past, Present and Future. Lexicography and Natural Language Processing (Euralex 2002), 1-30.

Busa, F. (1997). The Semantics of Agentive Nominals in the Generative Lexicon. In Saint-Dizier, P. (éd.), Predicative Forms in Natural Language. Amsterdam: Kluwer, 349-374

Cartoni B. \& Lefer M.-A. (2011) Negation and lexical morphology across languages: insights from a trilingual translation corpus. In Fernandez-Dominguez J., Lefer, M.A. et Renner V. (éds), Poznan Studies in Contemporary Linguistics. Special issue on 'English Word-Formation in Contrast.

Corbin, D. (2000). French (Indo-European: Romance). In Booij, G., Lehmann, Ch., et Mugdan, J. (éds.), An International Handbook on Inflection and Word-Formation / Ein internationales Handbuch zur Flexion und Wortbildung, New York: Mouton - Walter de Gruyter, 1285-1299.

Cruse, D. A. (1973). Some thoughts on agentivity. Journal of Linguistics 9, 11-23.

Dal, G., \& Amiot, D. (2008). La composition néoclassique en français et ordre des constituants. In Amiot, D. (éd.) $L a$ composition dans une perspective typologique. Arras: Artois Presse Université, 89-113.

Devos, F. \& Taeldeman J. (2004). Deverbal Nouns and the Agentive Dimension across Languages. In Willems, J., Defrancq, B., Colleman, T. \& Noël, D. (éds.) Contrastive Analysis in Language. Identifying Linguistic Units of Comparison, Palgrave Macmillan, 155-171.

Fisiak, J. (1983). Present trends in contrastive linguistics. In Sajavaara K. (éd.) Cross-language analysis and second language acquisition 1, Jyväskylä: University of Jyväskylä, 9-38.

Flaux, N. \& Van de Velde, D. (2000). Les noms en français : esquisse de classement. Paris : Ophrys.

Fradin, B. \& Kerleroux, F. (2003). Troubles with lexemes. 3d Mediterranean Morphology Meeting (MMM3) (selected papers), Barcelona, 177-196.

Fradin B., Dal G., Grabar N., Namer F., Lignon S., Tribout D. \& Zweigenbaum P. (2008). Remarques sur l'usage des corpus en morphologie. Langages 171, 34-59.

Fradin B., (2009) IE, Romance: French. In Lieber R. et Štekauer P. (éds.), The Oxford Handbook of Compounding, Oxford: Oxford University Press, 417-435.

Gross G. (1994). Classes d'objets et description des verbes. Langages 115, 15-30.

Gross G. (2011). Classification sémantique des noms humains collectifs, Cahiers de Lexicologie 98, 65-82.

Grossman M. \& F. Rainer (éds) (2004). La Formazione delle parole in italiano. Tubingen : Max Niemeyer Verlag.

Hathout N., Namer F., Plénat M. \& Tanguy L. (2009). La collecte et l'utilisation des données en morphologie. In Fradin B., F. Kerleroux and Plénat M. (éds.), Aperçus de Morphologie du français, Paris : Presses Universitaires de Vincennes, 267-287. 
James, C. (1980). Contrastive analysis. Burnt Mill : Longman Harlow.

Johansson, S. (2007). Seeing through multilingual corpora: on the use of corpora in contrastive studies. Amsterdam/Philadelphia : John Benjamins.

Lefer M.-A. (2011) Contrastive word-formation today: Retrospect and prospect. Poznan Studies in Contemporary Linguistics, 47(4), 645-682.

Lefer, M-A. \& Cartoni, B. (2011). Prefixes in contrast: Towards a meaning-based contrastive methodology for lexical morphology. In Bernardini S. et Hasselgård H. (éds.), Languages in Contrast, Amsterdam/Philadelphia: John Benjamins, 1-11.

Lignon S. \& Plénat M. (2009). Échangisme suffixal et contraintes phonologiques. In Fradin, B., Kerleroux, F. et Plénat, M. (éds), Aperçus de Morphologie du français, Paris: Presses Universitaires de Vincennes, 65-82

Lo Duca M. G. (2004). Nomi di agente. In Grossman M. et Rainer, F. (éds.), La Formazione delle parole in italiano, Tubingen : Max Niemeyer Verlag, 191-215.

Namer F. \& Villoing F. (2007). Have cutthroats anything to do with tracheotomes? Distinctive properties of VN vs NV compounds in French. Proceedings of the 5th Mediterranean Morphology Meeting (MMM5) Fréjus :105-124.

Roché M. (2011a). Quel traitement unifié pour les dérivations en -isme et en -iste. In Roché M., Boyé, G., Hathout, N., Lignon S. et Plénat, M. (éds.), Des Unités Morphologiques au Lexique. Paris : Hermès, 69-143.

Roché Michel. (2011b). Quelle morphologie ?, In Roché M., Boyé, G., Hathout, N., Lignon S. et Plénat, M. (éds.), Des Unités Morphologiques au Lexique. Paris : Hermès, 15-39.

\footnotetext{
${ }^{1}$ fuochista, construit sur fuoco (feu) désigne le spécialiste des chaudières à vapeur (centrales thermiques, locomotives à vapeur). chiusurista construit sur chiusura (fermeture) désigne le travailleur qui termine la journée de travail, ou le cycle de production.
} 